\title{
Effects of Sepsis on Neonatal Thrombopoiesis
}

\author{
RACHEL E. BROWN, LISA M. RIMSZA, KAREN PASTOS, LINDA YOUNG, MATTHEW A. SAXONHOUSE, \\ MATTHEW BAILEY, ROBERT M. LAWRENCE, AND MARTHA C. SOLA-VISNER
}

\author{
Department of Pediatrics [R.E.B., K.P., M.A.S., M.B., R.M.L., M.C.S.-V.], Department of Statistics [L.Y.], University of Florida, Gainesville, \\ Florida 32610; Department of Pathology [L.M.R.], University of Arizona, Tucson, Arizona 85721
}

\begin{abstract}
We serially evaluated the effects of sepsis and/or necrotizing enterocolitis (NEC) on neonatal thrombopoiesis, using a panel of tests that included platelet counts, thrombopoietin concentrations (Tpo), circulating megakaryocyte progenitor concentrations (CMPs), and reticulated platelets (RPs). Variables analyzed included sepsis type, time after onset of sepsis, platelet counts, and gestational (GA) and postconceptional ages (PCA). Twenty neonates were enrolled. Ten had Gram-negative, six had Gram-positive, and four had presumed sepsis. Four neonates had NEC stage II or higher, and six developed thrombocytopenia. Overall, septic neonates had significantly elevated Tpo concentrations and circulating megakaryocyte progenitors. The highest Tpo levels were associated with Gramnegative or presumed sepsis. RP percentages were increased only in neonates with low platelet counts, while RP counts $(\mathrm{RP} \% \times$ platelet count) were elevated in neonates with high platelet counts. Our findings suggest that septic neonates up-regulate Tpo production, leading to increased megakaryocytopoiesis and platelet release, although the degree of upregulation is moderate. The changes in RP\% and RP count most likely reflect increased thrombopoiesis with variable degrees of platelet consumption. In addition, our findings suggest that different factors, likely including level of illness and/or specific platelet or bacterial products, can down-regulate the magnitude of the thrombopoietic response. (Pediatr Res 64: 399-404, 2008)
\end{abstract}

$\mathrm{T}$ Thrombocytopenia is one of the most frequent hematologic abnormalities in the neonatal period, affecting $18-35 \%$ of all patients admitted to the Neonatal Intensive Care Unit $(1,2)$, and up to $73 \%$ of extremely low birth weight infants (birth weight $\leq 1000 \mathrm{~g}$ ) (3). In approximately $20-25 \%$ of these cases, the platelet count reaches a level believed to significantly increase the risk for hemorrhage (platelet count $<50,000 / \mu \mathrm{L}$ ) (4), with sepsis and necrotizing enterocolitis (NEC) being among the most common causes of severe neonatal thrombocytopenia. Treatment with platelet transfusion(s) is frequently provided to these infants in an attempt to diminish the occurrence and severity of hemorrhages. However, platelet transfusions carry several risks, including anaphylaxis, graft-versus-host disease, transmission of infection, and transfusion associated lung injury.

Because of these risks, thrombopoietic growth factors (i.e. thrombopoietin mimetics) are being investigated as alterna-

Received October 16, 2007; accepted May 6, 2008.

Correspondence: Rachel Brown, M.D., Department of Pediatrics, Division of Neonatology, Nationwide Children's Hospital, 722 Children's Drive, Columbus, OH 43205; e-mail: rachel.brown@nationwidechildrens.org

Supported by grants from the Children's Miracle Network (R.E.B.), American Heart Association Florida/Puerto Rico Affiliate (M.S.), and NIH Grant HL-69990 (M.S.V., L.R.). tives to platelet transfusions $(5,6)$. Based on the duration and severity of thrombocytopenia, neonates with sepsis and/or NEC could be potential candidates for therapy with thrombopoietic growth factors. However, it is unclear whether decreased platelet production contributes to their thrombocytopenia. In this regard, previous studies have reported elevated plasma thrombopoietin (Tpo) concentrations in neonates and children with sepsis $(7,8)$. The interpretation of these findings has been difficult, given the interplay of factors regulating circulating Tpo levels. Early studies of plasma Tpo concentrations concluded that hepatic Tpo production was constitutive, and that circulating Tpo levels were inversely regulated by the number of receptors (on hematopoietic progenitors, megakaryocytes, and platelets) available for binding $(9,10)$. Thus, elevated Tpo levels were considered synonymous with decreased megakaryocytes. More recent studies demonstrated that Tpo expression is up-regulated in inflammatory conditions, a response mostly mediated by IL-6 (11-13). Therefore, the significance of increased Tpo concentrations in neonatal sepsis is difficult to assess in the absence of other indicators of platelet production.

We designed this study to determine whether neonates upregulate Tpo production and thrombopoiesis during sepsis and/or NEC, or whether elevated Tpo levels that have been described are reflective of sepsis-associated bone marrow suppression, and therefore decreased numbers of receptors available for binding $(14,15)$. To answer this question, we used a panel of laboratory tests to evaluate platelet production using only peripheral blood samples. These tests included plasma thrombopoietin concentrations, concentrations of circulating megakaryocyte progenitors (CMPs), and reticulated platelets (RPs), which were compared with previously published normal ranges for neonates established in our laboratory (16-18).

Each one of these tests was designed to evaluate a different step in the process of platelet production. Specifically, Tpo is the most potent known stimulator of thrombopoiesis in the fetus, neonate, and adult (19-21). Plasma Tpo concentrations, measured by ELISA, reflect the balance between Tpo production and availability of Tpo receptors $(14,15)$. Megakaryocyte progenitors are cells that proliferate and generate large numbers of megakaryocytes. These progenitors can be identified by their ability to form megakaryocyte colonies when cultured

\footnotetext{
Abbreviations: CMP, Circulating megakaryocyte progenitor; GA, gestational age; NEC, necrotizing enterocolitis; PCA, postconceptional age; RP, reticulated platelet; TO, thiazole orange; Tpo, thrombopoietin
} 
Table 1. Mechanisms of thrombocytopenia according to the results of this panel

\begin{tabular}{|c|c|c|c|c|}
\hline Tpo & CMPs & $\mathrm{RP} \%$ & RP count & Interpretation \\
\hline$\downarrow$ to $\mathrm{NL}$ & $\downarrow$ & $\downarrow$ & $\downarrow \downarrow$ & $\begin{array}{l}\text { Decreased platelet production secondary to low Tpo levels: } \\
\text { Liver failure } \\
\text { Some anti-Tpo neutralizing antibodies }{ }^{38}\end{array}$ \\
\hline$\uparrow \uparrow \uparrow$ & $\downarrow$ & $\downarrow$ & $\downarrow \downarrow$ & $\begin{array}{l}\text { Decreased platelet production secondary to decreased marrow megakaryocytes: } \\
\text { CAMT }^{39} \\
\text { Osteopetrosis }{ }^{40} \\
\text { Aplastic anemia } \\
\text { Chemotherapy-induced thrombocytopenia } \\
\text { 27,10 }\end{array}$ \\
\hline$\uparrow$ & $\uparrow$ & $\downarrow$ & $\downarrow \downarrow$ & $\begin{array}{l}\text { Ineffective platelet production: } \\
\text { HIV infection }{ }^{42,43}\end{array}$ \\
\hline$\uparrow$ & $\uparrow$ & $\uparrow$ & $\downarrow$ to $\mathrm{NL}$ & $\begin{array}{l}\text { Thrombocytopenia due to increased platelet consumption with inflammation (Tpo up-regulation): } \\
\text { DIC }^{31} \\
\text { Sepsis? }\end{array}$ \\
\hline$\downarrow$ to $\mathrm{NL}$ & $\uparrow$ & $\uparrow$ & $\downarrow$ to $\mathrm{NL}$ & $\begin{array}{l}\text { Thrombocytopenia due to immune-mediated platelet consumption: } \\
\text { ITP } 27,10\end{array}$ \\
\hline
\end{tabular}

NL, normal; CAMT, congenital amegakaryocytic thrombocytopenia; HIV, Human Immunodeficiency Virus; ITP, Immune Thrombocytopenic Purpura; DIC, Disseminated Intravascular Coagulation.

in semisolid media with appropriate stimulatory cytokines. In neonates, megakaryocyte progenitors circulate in the blood, and their concentration is thought to reflect the concentration of bone marrow megakaryocytes and progenitors $(16,22,23)$. Reticulated platelets (RP) are newly produced platelets $(<24$-h old) that contain residual RNA (RNA) (24), which allows them to be detected and quantified in the blood using flow cytometry $(17,25)$. The reticulated platelet percentage is frequently used as a measure of platelet release, although some experts advocate the use of reticulated platelet counts $(\mathrm{RP} \% \times$ platelet count) as a more accurate parameter.

In combination, these tests allowed us to assess three of the four major steps of platelet production (production of the thrombopoietic stimulus, megakaryocyte proliferation, and platelet release). This panel of tests was also chosen based on a number of previous studies in children and adults, which demonstrated that the use of several tests in combination was better at differentiating disorders of production from disorders of consumption than any single test $(20,26,27)$. Specific mechanisms of thrombocytopenia that have been identified applying some or all of these tests, and the interpretation of different patterns, are summarized in Table 1 .

\section{PATIENTS AND METHODS}

Neonates admitted to the NICU at Shands Hospital at the University of Florida between November of 2003 and November of 2005 who were treated for confirmed or presumed sepsis were eligible for study. Confirmed sepsis was defined as a positive blood culture accompanied by clinical signs of infection and a full course of antibiotics, and presumed sepsis was defined using strict preset criteria (Table 2). Approval for this study was obtained from the University of Florida Institutional Review Board. Signed informed consent was obtained from the mothers of all neonates enrolled. After enrollment, patients underwent serial blood draws on d 3-4, 7-8, and 12-14 after the initial sepsis work up. These time points were chosen based on the literature, with the intention to capture the peak Tpo concentrations after the onset of sepsis (d 3-4), the peak response to an increase in Tpo (d 7-8), and the resolution of thrombocytopenia or the onset of thrombocytosis following infection (d 12-14). Platelet counts, plasma Tpo concentrations, CMPs, and RPs were measured at each time point. For the purposes of this study, thrombocytopenia was defined a priori as a platelet count $<100,000 / \mu \mathrm{L}$.

Nonthrombocytopenic healthy neonates enrolled in three previously published studies carried out by our group established reference ranges for the tests applied in the present study. Briefly, plasma Tpo concentrations were measured in 47 nonthrombocytopenic neonates with a mean gestational age
(GA) of $35 \pm 5 \mathrm{wk}$ (range 26-42). Their median Tpo concentration was 93 $\mathrm{pg} / \mathrm{mL}$, and the mean was $81.4 \pm 8.4$ (18). Forty-two nonthrombocytopenic neonates were studied to establish the normal concentration of CMPs. The mean GA of these infants was $31 \pm 5$ wk (range 25-41) and their mean postconceptional age (PCA, gestational age + weeks of life) was $33 \pm 4.8 \mathrm{wk}$ (range 25-42). The mean CMP concentration was $99.5 \pm 13.6$ (16). Normal $\mathrm{RP} \%$ were established in 37 nonthrombocytopenic neonates with a mean gestational age of $31 \pm 4.5 \mathrm{wk}$ (range 25-41) and a PCA of $33 \pm 4.2 \mathrm{wk}$ (range 27-41). The mean RP\% was $2.6 \pm 1.4 \%$ (17).

Blood $(1.6 \mathrm{~mL}$ ), obtained from a venous or arterial central line (when available) or by venipuncture, was immediately placed in a citrated tube and processed.

Table 2. Preestablished criteria for presumed sepsis

CBC with an I/T ratio* $>20 \%$ or and acute platelet drop to $\leq 100,000 / \mu \mathrm{L}$ AND

Antibiotic and/or antifungal therapy will be continued for $\geq 72$ hours AND

Patient has at least one clinical sign or symptom from each of $\geq 2$ of the defined categories listed below in the preceding 48 hours

Respiratory

Apnea (defined as cessation of breathing for $>20$ seconds or cessation of breathing that results in cyanosis or bradycardia)

Unexplained tachypnea (defined as respiratory rate $>60$ breaths/minute)

Unexplained nasal flaring, retractions or grunting $\dagger$

Unexplained increase in the oxygen requirement $\dagger$

Cardiovascular

Bradycardia (defined as a heart rate $<100$ beats/minute in premature infants or $<80$ beats/minute in term infants) or tachycardia (heart rate $>180$ beats/minute)

Hypotension requiring intervention

Poor peripheral perfusion $\dagger$

Neurologic

Lethargy $\dagger$

Irritability $\dagger$

Seizures

Gastrointestinal

Abdominal distension $\dagger$

Feeding intolerance (defined as emesis or increased gastric residuals) $\dagger$

Autonomic

Fever (defined as central temperature $\geq 38^{\circ} \mathrm{C}$ measured twice, at least one hour apart)

Temperature instability $\dagger$

* I/T ratio is the number of immature neutrophil cells divided by the total neutrophil number.

$\dagger$ Deemed significant by the attending physician. 
Platelet counts. Complete blood counts with platelets were obtained using a Coulter AcT10 cell counter (Beckman Coulter, Inc., Fullerton, CA).

Plasma Tpo concentrations. Blood was spun at $1000 \mathrm{G}$ for $30 \mathrm{~min}$. The supernatant was then transferred to a new microfuge tube and spun at $10,000 \mathrm{~g}$ for $10 \mathrm{~min}$. The remaining supernatant (platelet-poor plasma) was stored at $\leq-70^{\circ} \mathrm{C}$ until analysis. Plasma Tpo concentrations were measured using a commercially available ELISA (R\&D Systems, Minneapolis, MN) with a lower detection limit of $15 \mathrm{pg} / \mathrm{mL}$.

Circulating megakaryocyte progenitor assay. CMP assays were carried out using our previously published methods (16). Briefly, light density mononuclear cells, isolated from whole blood using gradient centrifugation, were cultured in a serum-free, semisolid growth medium (MegaCult ${ }^{\circledR}$, StemCell Technologies, Vancouver) with 50 ng/mL rhTpo (Peprotech, Rocky Hill, $\mathrm{NJ})$. Cells were plated in double-chamber slides at a final density of $5 \times 10^{5}$ light density mononuclear cells per slide. After 10-12 d, the slides were dehydrated, fixed, and immunohistochemically stained with a mouse antihuman GPIIb/IIIa antibody (MegaCult staining kit, StemCell Technologies, Vancouver). Megakaryocyte colonies were then counted using a standard microscope. Results were expressed as number of megakaryocyte colonies per $5 \times 10^{5}$ light density mononuclear cells plated.

Reticulated platelets. Reticulated platelets were quantified following our previously described method (17). In brief, whole citrated blood was fixed overnight, incubated with and without RNase (Sigma Chemical Co., St. Louis, MO) and stained with an anti-CD41 antibody and with thiazole orange (TO; ReticCount, Becton Dickinson, Franklin Lakes, NJ), which stains RNA. Two-color flow cytometry (FacScan, Becton Dickinson, Franklin Lakes, NJ) was used to detect the CD41 antigen on the surface of the platelets and their RNA content. The RNase treated sample was used to correct for nonspecific staining of platelet granules by TO. Thus, the RP\% was calculated by the following equation:

[NonRNase treated $\mathrm{CD} 41^{+} \mathrm{TO}^{+}$events - RNase treated $\mathrm{CD} 41^{+} \mathrm{TO}^{+}$events]

\section{Total number of $\mathrm{CD} 41^{+}$events}

The reticulated platelet count, which represents the absolute concentration of reticulated platelets in the blood, was also calculated by multiplying the RP\% by the platelet count.

Statistical analysis. Before statistical analysis, platelet counts, thrombopoietin levels, and RPs were subjected to logarithmic transformation to better meet the assumption of normality. For these variables, statistical methods were applied on the transformed variables, and results were reported on the original scale. Circulating megakaryocyte progenitors were directly analyzed, without logarithmic transformation. The data were analyzed using a mixed model analysis, and GA, postconceptional age, and platelet count were treated as potential covariates. Comparisons between thrombocytopenic and non- thrombocytopenic infants were carried out using an ANOVA with repeated measures and a posthoc test. All results are presented as means \pm SEM.

\section{RESULTS}

A total of 20 neonates ( 12 males) were enrolled. Their mean GA was $30.1 \mathrm{wk}$ (range 24-40), and mean PCA was $35.5 \mathrm{wk}$ (range 28.5-48). Six patients had Gram-positive sepsis, 10 had Gram-negative sepsis, and four had presumed sepsis. Four neonates were diagnosed with NEC stage II or higher. Clinical characteristics of the study subjects are summarized in Table 3. Overall, patients with Gram-negative sepsis appeared sicker in response to the infection, compared with the other two groups. Four of the patients enrolled died before discharge, but none died during the study period.

Platelet counts. Six neonates developed thrombocytopenia (platelet count $<100,000 / \mu \mathrm{L}$ ), three with nadir platelet counts $<50,000 / \mu \mathrm{L}$. All three patients with platelet counts $<50,000 / \mu \mathrm{L}$ had Gram-negative sepsis. However, the mean platelet counts in the three sepsis groups were not statistically different from each other $(p=0.17)$. Other variables examined (GA, PCA, and NEC) did not have a significant effect on the platelet count.

Tpo concentrations. Overall, septic neonates had higher Tpo concentrations than those previously reported in healthy neonates (18) $(180.5 \pm 16.5 \mathrm{pg} / \mathrm{mL}$ versus $81.4 \pm 8.4 \mathrm{pg} / \mathrm{mL}$, respectively; $p<0.0001$ ), although the type of infection significantly influenced the magnitude of this increase. Specifically, patients with Gram-negative and presumed sepsis had higher Tpo concentrations than healthy neonates (146 \pm $24 \mathrm{pg} / \mathrm{mL}$ and $278 \pm 73 \mathrm{pg} / \mathrm{mL}$, respectively, versus $81.4 \pm$ $8.4 \mathrm{pg} / \mathrm{mL}$ in normal neonates; $p<0.05$ ). Tpo concentrations in patients with Gram-positive sepsis also tended to be higher than those of healthy newborn infants, although this difference

Table 3. Clinical characteristics of enrolled patients

\begin{tabular}{|c|c|c|c|c|c|c|c|c|}
\hline Patient & Sepsis category & Gender & GA (wks) & PCA (wks) & $\Delta$ Respiratory support $\dagger$ & $\Delta \mathrm{O}_{2}$ requirement* & NEC & $\downarrow \mathrm{PC}$ \\
\hline 1 & Gram-positive & M & 37 & 48 & $\mathrm{NCPAP} \rightarrow \mathrm{CMV}$ & $50 \% \rightarrow 60 \%$ & No & No \\
\hline 2 & Gram-positive & M & 37 & 40 & CMV & None & No & No \\
\hline 3 & Gram-positive & M & 24 & 37 & CMV & None & No & No \\
\hline 4 & Gram-positive & M & 39 & 40 & CMV & $40 \% \rightarrow 50 \%$ & No & No \\
\hline 5 & Gram-positive & $\mathrm{F}$ & 26 & 30 & CMV & $35 \% \rightarrow 50 \%$ & No & Yes \\
\hline 6 & Gram-positive & M & 26 & 29 & $\mathrm{NCPAP} \rightarrow \mathrm{CMV}$ & $31 \% \rightarrow 28 \%$ & No & No \\
\hline 7 & Gram-negative & $\mathrm{F}$ & 40 & 41 & $\mathrm{RA} \rightarrow \mathrm{CMV}$ & $21 \% \rightarrow 30 \%$ & Yes (surgical) & Yes \\
\hline 8 & Gram-negative & $\mathrm{F}$ & 28 & 30 & $\mathrm{RA} \rightarrow \mathrm{CMV}$ & $21 \% \rightarrow 54 \%$ & Yes (surgical) & Yes \\
\hline 9 & Gram-negative & M & 26 & 33 & $\mathrm{NCPAP} \rightarrow \mathrm{CMV}$ & $25 \% \rightarrow 30 \%$ & No & No \\
\hline 10 & Gram-negative & M & 25 & 33 & $\mathrm{NCPAP} \rightarrow \mathrm{HFOV}$ & $30 \% \rightarrow 100 \%$ & Yes (surgical) & Yes \\
\hline 11 & Gram-negative & $\mathrm{F}$ & 27 & 31 & CMV & $48 \% \rightarrow 90 \%$ & No & Yes \\
\hline 12 & Gram-negative & M & 28 & 33 & $\mathrm{NCPAP} \rightarrow \mathrm{CMV}$ & $25 \% \rightarrow 80 \%$ & No & No \\
\hline 13 & Gram-negative & $\mathrm{F}$ & 25 & 28 & CMV & None & No & No \\
\hline 14 & Gram-negative & $\mathrm{F}$ & 32 & 38 & $\mathrm{RA} \rightarrow \mathrm{CMV}$ & $21 \% \rightarrow 30 \%$ & No & Yes \\
\hline 15 & Gram-negative & $\mathrm{F}$ & 32 & 35 & NCPAP & None & No & No \\
\hline 16 & Gram-negative & M & 34 & 37 & $\mathrm{RA} \rightarrow \mathrm{CMV}$ & $21 \% \rightarrow 30 \%$ & No & No \\
\hline 17 & Presumed & M & 28 & 48 & $\mathrm{NC} \rightarrow \mathrm{CMV}$ & $0.2 \mathrm{~L} \rightarrow 100 \%$ & No & No \\
\hline 18 & Presumed & M & 26 & 32 & CMV & None & No & No \\
\hline 19 & Presumed & M & 32 & 32 & CMV & $100 \% \rightarrow 40 \%$ & No & No \\
\hline 20 & Presumed & $\mathrm{F}$ & 30 & 33 & $\mathrm{NC}$ & $0.1 \mathrm{~L} \rightarrow 0.2 \mathrm{~L}$ & Yes & No \\
\hline
\end{tabular}

$\dagger$ Level of support required $24^{\circ}$ prior to sepsis evaluation $\rightarrow$ highest level of support within $72^{\circ}$ after evaluation.

* Oxygen requirement $24^{\circ}$ prior to sepsis evaluation $\rightarrow$ highest oxygen requirement within $72^{\circ}$ after evaluation.

GA, Gestational age; PCA, Post-conceptional age; NCPAP, Nasal Continuous Positive Airway Pressure; CMV, Conventional Mechanical Ventilation; RA, Room Air; HFOV, High-frequency Oscillatory Ventilation; NC, Nasal Cannula; NEC, Necrotizing Enterocolitis; PC, Platelet Count. 
was not statistically significant (137.6 $\pm 32 \mathrm{pg} / \mathrm{mL}$ compared with $81.4 \pm 8.4 \mathrm{pg} / \mathrm{mL}$; NS).

Gestational age and postconceptional age did not significantly correlate with plasma Tpo concentrations, and neither did the diagnosis of NEC. However, Tpo decreased as the platelet count increased, with a negative coefficient of -0.0007 ( $p=0.006)$.

Circulating megakaryocyte progenitors (CMPs). Overall, septic neonates had significantly higher concentrations of circulating megakaryocyte progenitors than healthy neonates (16) $(202.1 \pm 20.4$ versus $99.5 \pm 13.6$, respectively; $p<$ $0.0001)$. The time course of this increase was different depending on the type of infection (Fig. 1). At the initial time point (d 3-4 after onset of infection), CMPs were significantly increased in neonates with Gram-negative and Gram-positive infections (182.5 \pm 27.6 and $308.4 \pm 46.4$, respectively). Patients with presumed sepsis had CMPs within normal limits at that time point $(60.8 \pm 42.4)$, but these increased by $7-8 \mathrm{~d}$ after the onset of sepsis (254 \pm 97.1). At 10-14 d after infection, the concentration of CMPs was within normal limits in the Gram-positive and presumed sepsis group, but was still elevated in the Gram-negative group (187.9 \pm 39.9$)$.

Among the other co-variables examined, the PCA was inversely correlated with circulating megakaryocyte progenitors, with a coefficient of -7.5 , implying that the concentration of circulating megakaryocyte progenitors decreased by an average of 7.5 for every week of life, while controlling for all the other covariates. The platelet count and the diagnosis of NEC did not have significant effects on circulating megakaryocyte progenitor concentration.

Reticulated platelets. Unlike with the previous two measures of thrombopoiesis, the mean RP\% in septic neonates was not different from that in healthy neonates (17). Specifically, the mean RP\% was $2.7 \pm 0.45 \%$ in neonates with Gramnegative sepsis, $1.68 \pm 0.38 \%$ in neonates with Gram-positive sepsis, and $2.34 \pm 0.65 \%$ in those with presumed sepsis, all of

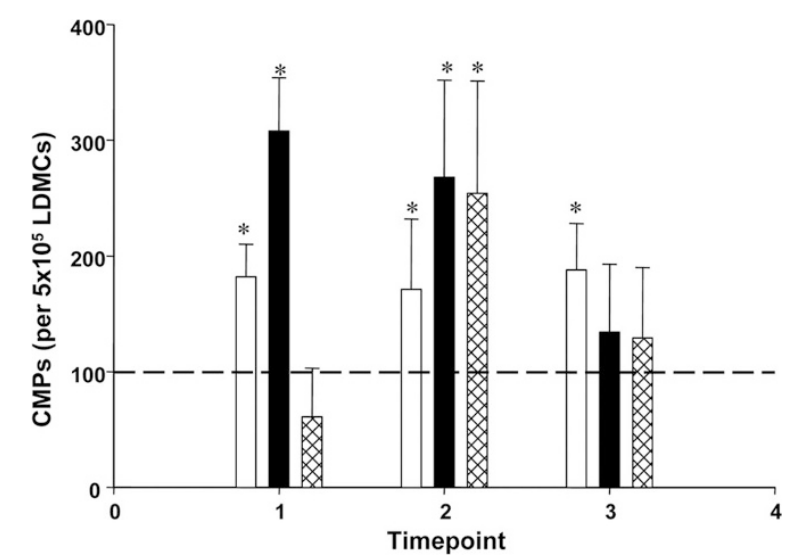

Figure 1. Circulating megakaryocyte progenitors according to type of sepsis and time after onset. Septic neonates had increased CMPs compared with healthy neonates. However, the concentration of CMPs varied depending on the type of sepsis (Gram-negative, $\square$; Gram-positive, $\mathbf{\square}$; or presumed, $\mathbf{X}$ ) and the time after onset of sepsis. Time points 1,2, and 3 represent data collected $3-4,7-8$, and $12-14 \mathrm{~d}$ after the onset of sepsis, respectively. The horizontal line represents the mean concentration of CMPs for healthy neonates (99.5/ $5 \times 10^{5}$ LDMCs). The asterisk indicates statistical significance $(p<0.05)$ compared with healthy infants. them comparable to our previously reported mean RP\% of $2.6 \pm$ $1.4 \%$ in normal neonates (17). Other variables examined (i.e. GA, PCA, and NEC) did not have a significant effect on RP\%. However, there was a significant inverse correlation between RP $\%$ and platelet count $(r=-0.43, p=0.002$; Fig. $2 A)$.

As with the RP\% we found no difference in RP count between neonates with and without sepsis. Neither the presence of NEC nor thrombocytopenia significantly correlated with the RP count. However, there was a significant positive correlation between RP count and platelet count, indicating that higher platelet counts were associated with higher RP counts $(p=0.001$; Fig. $2 B)$.

\section{DISCUSSION}

Sepsis and necrotizing enterocolitis in neonates are frequently accompanied by thrombocytopenia. However, the exact mechanisms underlying the thrombocytopenia in these conditions remain unclear, and it is not known whether septic neonates have increased or decreased platelet production, and if so, at what step the process of thrombopoiesis is affected. This study was designed to address this question by evaluating the status of platelet production in septic neonates using a panel of tests reflecting different steps in thrombopoiesis.
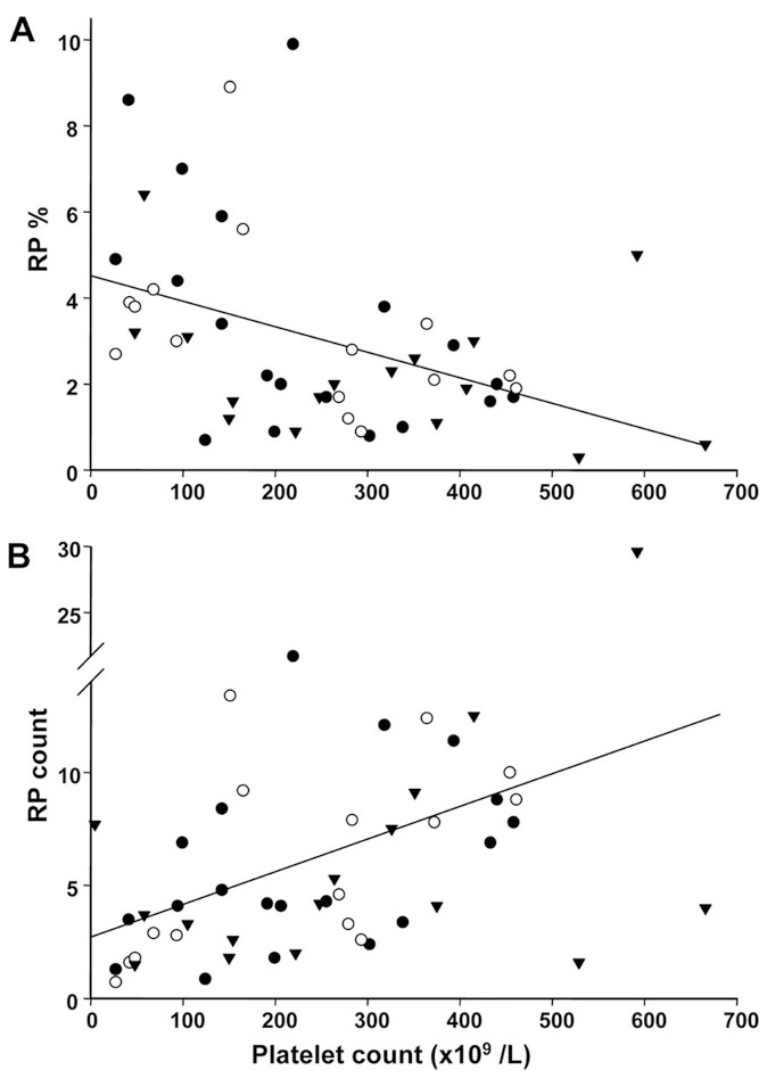

Figure 2. (A). Platelet count and $\mathrm{RP} \%$. RP\% were significantly elevated in neonates with thrombocytopenia (platelet counts $<100,000 / \mu \mathrm{L}$ ). There was a statistically significant inverse linear correlation between RP\% and platelet counts $(p=0.002)$. (B) Platelet count and absolute RP count. Contrary to $\mathrm{RP} \%$, absolute RP counts increased with increasing platelet counts. There was a significant correlation between RP counts and platelet counts $(p=0.001)$. Data collected 3-4 d after onset of sepsis (-);values obtained at 7-8 d $(\bigcirc)$;values 12-14 d after sepsis onset $(\mathbf{\nabla})$. 
Consistent with other studies in neonates and children with infection $(7,8,11)$, septic neonates in our study had elevated plasma Tpo concentrations, compared with healthy neonates (18). However, this elevation was mostly evident in neonates with presumed sepsis and was only moderate in patients with Gram-negative and Gram-positive sepsis. Neonates with Gram-negative sepsis appeared to be sicker than those in the other groups, thus contradicting findings in adults, in whom the severity of sepsis has been reported to be the major determinant of circulating Tpo levels (28). In this regard, previous studies have suggested that neonates might have developmental limitations in their ability to up-regulate Tpo production compared with adults $(18,29)$, although the influence of level of illness or specific bacterial products on these findings is unknown. Significant interindividual differences in the response to stress were also observed, as exemplified by patient \# 18 (Table 3), who had an initial Tpo concentration of $1268 \mathrm{pg} / \mathrm{mL}$ (the highest in our study), which 2 wk later presumably led to a platelet count of $948,000 / \mu \mathrm{L}$. The presence of this patient in the presumed sepsis group, combined with the small sample size of that group $(n=4)$, may have contributed to the observation that neonates with presumed sepsis had the highest Tpo concentrations.

Among other factors investigated, we also found that the platelet count had a significant inverse association with Tpo concentration. A correlation between platelet counts and Tpo concentrations has been previously found by some investigators $(7,11,28)$, but not by others $(8,30,31)$, likely reflecting the fact that the platelet count is one of several factors that determine the ultimate circulating Tpo concentration in patients with infections.

Circulating megakaryocyte progenitors have been used as an indirect measure of megakaryocytopoiesis in neonates, based on the demonstration of a correlation between circulating and marrow megakaryocyte progenitors in children (22). In neonates, we have previously demonstrated that CMPs also decrease with increasing postconceptional age, a factor that has to be taken into account when interpreting the results of this assay (16). As in our previous study in healthy neonates, we again found an inverse correlation between PCA and concentration of CMPs in this cohort of septic infants.

Overall, our current study revealed increased concentrations of CMPs in septic neonates compared with their healthy growing counterparts, suggesting that Tpo elevations are accompanied by increased megakaryocytopoiesis. Among the variables examined, the type of infection and the time after the onset of infection had the most significant effects on the concentration of CMPs. Neonates with Gram-negative infections, the group with the highest incidence of thrombocytopenia, had the least pronounced (although most persistent) elevations in CMPs. While the explanation for this finding is unclear, it is possible that their increased severity of illness led to some degree of suppression of megakaryocytopoiesis. This explanation is consistent with our previous findings of decreased megakaryocytes in postmortem bone marrow specimens compared with marrow samples from living neonates (32). A number of mechanisms could contribute to these observations, including the relatively modest levels of Tpo up-regulation or high levels of platelet factor 4 being released from activated platelets during severe sepsis. It has been recently demonstrated that platelet factor 4 is a negative regulator of megakaryocytopoiesis, inhibiting megakaryocyte proliferation (33). Alternatively, it is possible that specific bacterial products released from Gram-negative organisms suppressed megakaryocyte proliferation.

Among the parameters studied, the RP\% was the only one that was not significantly increased in septic compared with normal neonates. Neither type of infection nor time after onset of infection had a significant effect on the RP\%. However, there was a significant inverse correlation between platelet count and RP\%. These observations are consistent with a previous study in healthy adults, in which infusion of endotoxin induced an abrupt decrease in platelet counts, followed by a rapid rise in RP\% (34).

To determine whether a high RP\% translated into an absolute increased concentration of newly released platelets, we evaluated RP counts. Increased RP counts were observed mostly in neonates with high platelet counts, but not in infants with thrombocytopenia. Although the mechanisms underlying these observations are unknown, this constellation of findings can be explained by the model proposed in Figure 3. According to this model, both platelet production and platelet consumption are increased in neonates with sepsis, but there is variability in the degree to which these processes are affected in individual patients at any given time point, thus leading to the three clinical scenarios proposed. As evidenced in Figures $2 A$ and $B$, measurements obtained at times when platelet consumption exceeded platelet production were characterized by low platelet counts, high RP\%, and low to normal RP counts. If platelet production and consumption were equilibrated, all three parameters were within normal limits. However, if the rate of platelet production exceeded the rate of platelet consumption, the platelet count and the absolute RP counts increased, while the RP\% remained close to normal. The latter case scenario is consistent with observations in adult patients with immune thrombocytopenic purpura, in whom treatment with a thrombopoietin mimetic increased platelet counts and absolute immature platelet fractions (the clinical equivalent of a reticulated platelet count), while the immature platelet percentage remained relatively unchanged (Psaila B et al. American Society of Hematology, December 9-12, 2006, Orlando, FL).

In summary, we recognize the limitations of our study, including the small sample size and the lack of direct or

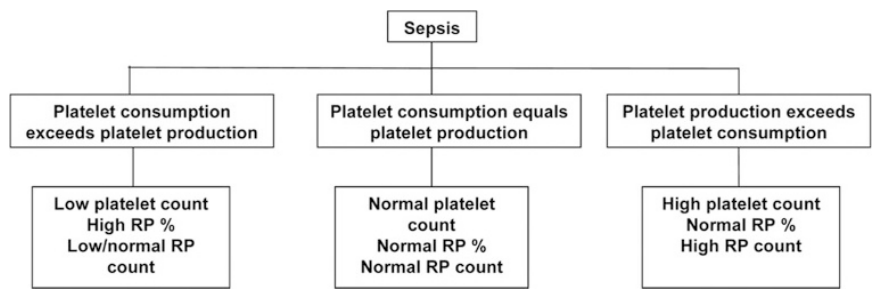

Figure 3. Proposed model to explain the changes in RP\% and RP count according to platelet count. According to our model, both platelet production and platelet consumption are increased during sepsis, but to variable degrees at different time points. This variability leads to the three proposed scenarios. 
quantitative measurements of platelet consumption or marrow megakaryocytes. However, we believe our findings provide new insights into the mechanisms underlying the thrombocytopenia of neonatal sepsis. Specifically, our observations strongly suggest that neonates respond to sepsis by upregulating Tpo production and thrombopoiesis, although the degree of up-regulation is modest. Thrombocytopenia ensues when the rate of platelet consumption exceeds the rate of platelet production. In addition, our findings suggest that severe illness, or alternatively specific platelet or bacterial products, down-regulate the magnitude of the thrombopoietic response, and can lead to a state of "relative hypoproliferation," previously defined as a less than twofold increase in megakaryocytopoiesis in response to thrombocytopenia (35). It remains to be determined whether the response of neonates to increased platelet demand (in sepsis or in other processes) is quantitatively similar to that of adults with similar pathophysiologic processes, or whether the recently described developmental differences in the ability of neonates to increase megakaryocyte size (32) and Tpo production further limit their ability to up-regulate platelet production. If so, the potential effects of therapy with thrombopoietin agents would warrant further study in this population.

\section{REFERENCES}

1. Mehta P, Vasa R, Neumann L, Karpatkin M 1980 Thrombocytopenia in the high-risk infant. J Pediatr 97:791-794

2. Castle V, Andrew M, Kelton J, Giron D, Johnston M, Carter C 1986 Frequency and mechanism of neonatal thrombocytopenia. J Pediatr 108:749-755

3. Christensen RD, Henry E, Wiedmeier SE, Stoddard RA, Sola-Visner MC, Lambert DK, Kiehn TI, Ainsworth S 2006 Thrombocytopenia among extremely low birth weight neonates: data from a multihospital healthcare system. J Perinatol 26:348353

4. Andrew M, Castle V, Saigal S, Carter C, Kelton JG 1987 Clinical impact of neonatal thrombocytopenia. J Pediatr 110:457-464

5. Tsimberidou AM, Giles FJ, Khouri I, Bueso-Ramos C, Pilat S, Thomas DA, Cortes J, Kurzrock R 2005 Low-dose interleukin-11 in patients with bone marrow failure: update of the M. D. Anderson Cancer Center experience. Ann Oncol 16:139-145

6. Bussel JB, Kuter DJ, George JN, McMillan R, Aledort LM, Conklin GT, Lichtin AE, Lyons RM, Nieva J, Wasser JS, Wiznitzer I, Kelly R, Chen CF, Nichol JL 2006 AMG 531, a thrombopoiesis-stimulating protein, for chronic ITP. N Engl J Med 355:1672-1681

7. Colarizi P, Fiorucci P, Caradonna A, Ficuccilli F, Mancuso M, Papoff P 1999 Circulating thrombopoietin levels in neonates with infection. Acta Paediatr 88:332337

8. Oygur N, Tunga M, Mumcu Y, Yesilipek A, Gura A, Coskun M, Yegin O 2001 Thrombopoietin levels of thrombocytopenic term and preterm newborns with infection. Am J Perinatol 18:279-286

9. Fielder PJ, Gurney AL, Stefanich E, Marian M, Moore MW, Carver-Moore K, de Sauvage FJ 1996 Regulation of thrombopoietin levels by c-mpl-mediated binding to platelets. Blood 87:2154-2161

10. Emmons RV, Reid DM, Cohen RL, Meng G, Young NS, Dunbar CE, Shulman NR 1996 Human thrombopoietin levels are high when thrombocytopenia is due to megakaryocyte deficiency and low when due to increased platelet destruction. Blood 87:4068-4071

11. Ishiguro A, Suzuki Y, Mito M, Shimbo T, Matsubara K, Kato T, Miyazaki H 2002 Elevation of serum thrombopoietin precedes thrombocytosis in acute infections. Br J Haematol 116:612-618

12. Wolber EM, Fandrey J, Frackowski U, Jelkmann W 2001 Hepatic thrombopoietin mRNA is increased in acute inflammation. Thromb Haemost 86:1421-1424

13. Wolber EM, Jelkmann W 2000 Interleukin-6 increases thrombopoietin production in human hepatoma cells HepG2 and Hep3B. J Interferon Cytokine Res 20:499-506

14. Kaushansky K 2005 The molecular mechanisms that control thrombopoiesis. J Clin Invest 115:3339-3347

15. Kaushansky K 2006 Lineage-specific hematopoietic growth factors. N Engl J Med 354:2034-2045

16. Saxonhouse MA, Christensen RD, Walker DM, Hutson AD, Sola MC 2004 The concentration of circulating megakaryocyte progenitors in preterm neonates is a function of post-conceptional age. Early Hum Dev 78:119-124
17. Saxonhouse MA, Sola MC, Pastos KM, Ignatz ME, Hutson AD, Christensen RD, Rimsza LM 2004 Reticulated platelet percentages in term and preterm neonates. J Pediatr Hematol Oncol 26:797-802

18. Sola MC, Calhoun DA, Hutson AD, Christensen RD 1999 Plasma thrombopoietin concentrations in thrombocytopenic and non-thrombocytopenic patients in a neonatal intensive care unit. Br J Haematol 104:90-92

19. Kaushansky K, Broudy VC, Lin N, Jorgensen MJ, McCarty J, Fox N, ZuckerFranklin D, Lofton-Day C 1995 Thrombopoietin, the Mp1 ligand, is essential for full megakaryocyte development. Proc Natl Acad Sci USA 92:3234-3238

20. Murray NA, Watts TL, Roberts IA 1998 Endogenous thrombopoietin levels and effect of recombinant human thrombopoietin on megakaryocyte precursors in term and preterm babies. Pediatr Res 43:148-151

21. Sola MC, Du Y, Hutson AD, Christensen RD 2000 Dose-response relationship of megakaryocyte progenitors from the bone marrow of thrombocytopenic and nonthrombocytopenic neonates to recombinant thrombopoietin. $\mathrm{Br} \mathrm{J}$ Haematol 110:449-453

22. Murray NA, Roberts IA 1995 Circulating megakaryocytes and their progenitors (BFU-MK and CFU-MK) in term and pre-term neonates. Br J Haematol 89:41-46

23. Murray NA, Roberts IA 1996 Circulating megakaryocytes and their progenitors in early thrombocytopenia in preterm neonates. Pediatr Res 40:112-119

24. Ault KA, Knowles C 1995 In vivo biotinylation demonstrates that reticulated platelets are the youngest platelets in circulation. Exp Hematol 23:996-1001

25. Peterec SM, Brennan SA, Rinder HM, Wnek JL, Beardsley DS 1996 Reticulated platelet values in normal and thrombocytopenic neonates. J Pediatr 129:269-274

26. Fabris F, Cordiano I, Steffan A, Ramon R, Scandellari R, Nichol JL, Girolami A 2000 Indirect study of thrombopoiesis (TPO, reticulated platelets, glycocalicin) in patients with hereditary macrothrombocytopenia. Eur J Haematol 64:151-156

27. Kurata Y, Hayashi S, Kiyoi T, Kosugi S, Kashiwagi H, Honda S, Tomiyama Y 2001 Diagnostic value of tests for reticulated platelets, plasma glycocalicin, and thrombopoietin levels for discriminating between hyperdestructive and hypoplastic thrombocytopenia. Am J Clin Pathol 115:656-664

28. Zakynthinos SG, Papanikolaou S, Theodoridis T, Zakynthinos EG, ChristopoulouKokkinou V, Katsaris G, Mavrommatis AC 2004 Sepsis severity is the major determinant of circulating thrombopoietin levels in septic patients. Crit Care Med 32:1004-1010

29. Watts TL, Murray NA, Roberts IA 1999 Thrombopoietin has a primary role in the regulation of platelet production in preterm babies. Pediatr Res 46:28-32

30. Bjerre A, Ovstebo R, Kierulf P, Halvorsen S, Brandtzaeg P 2000 Fulminant meningococcal septicemia: dissociation between plasma thrombopoietin levels and platelet counts. Clin Infect Dis 30:643-647

31. Hiyoyama K, Wada H, Shimura M, Nakasaki T, Katayama N, Nishikawa M, Shiku H, Tahara T, Kato T 1997 Increased serum levels of thrombopoietin in patients with thrombotic thrombocytopenic purpura, idiopathic thrombocytopenic purpura, or disseminated intravascular coagulation. Blood Coagul Fibrinolysis 8:345-349

32. Sola-Visner MC, Christensen RD, Hutson AD, Rimsza LM 2007 Megakaryocyte size and concentration in the bone marrow of thrombocytopenic and nonthrombocytopenic neonates. Pediatr Res 61:479-484

33. Lambert MP, Rauova L, Bailey M, Sola-Visner MC, Kowalska MA, Poncz M 2007 Platelet factor 4 is a negative autocrine in vivo regulator of megakaryopoiesis: clinical and therapeutic implications. Blood 110:1153-1160

34. Stohlawetz P, Folman CC, von dem Borne AE, Pernerstorfer T, Eichler HG, Panzer S, Jilma B 1999 Effects of endotoxemia on thrombopoiesis in men. Thromb Haemost 81:613-617

35. Harker LA, Finch CA 1969 Thrombokinetics in man. J Clin Invest 48:963-974

36. Peck-Radosavljevic M, Wichlas M, Zacherl J, Stiegler G, Stohlawetz P, Fuchsjager M, Kreil A, Metz-Schimmerl S, Panzer S, Steininger R, Muhlbacher F, Ferenci P, Pidlich J, Gangl A 2000 Thrombopoietin induces rapid resolution of thrombocytopenia after orthotopic liver transplantation through increased platelet production. Blood 95:795-801

37. Panasiuk A, Prokopowicz D, Zak J, Panasiuk B 2004 Reticulated platelets as a marker of megakaryopoiesis in liver cirrhosis; relation to thrombopoietin and hepatocyte growth factor serum concentration. Hepatogastroenterology 51:11241128

38. Li J, Yang C, Xia Y, Bertino A, Glaspy J, Roberts M, Kuter DJ 2001 Thrombocytopenia caused by the development of antibodies to thrombopoietin. Blood 98:32413248

39. Savoia A, Dufour C, Locatelli F, Noris P, Ambaglio C, Rosti V, Zecca M, Ferrari S, di Bari F, Corcione A, Di Stazio M, Seri M, Balduini CL 2007 Congenital amegakaryocytic thrombocytopenia: clinical and biological consequences of five novel mutations. Haematologica 92:1186-1193

40. Colarizi P, Fiorucci P, Roggini M, Papoff P 1999 Circulating thrombopoietin levels in a neonate with osteopetrosis. Pediatrics 103:700-701

41. Marsh JC, Gibson FM, Prue RL, Bowen A, Dunn VT, Hornkohl AC, Nichol JL, Gordon-Smith EC 1996 Serum thrombopoietin levels in patients with aplastic anaemia. Br J Haematol 95:605-610

42. Cole JL, Marzec UM, Gunthel CJ, Karpatkin S, Worford L, Sundell IB, Lennox JL, Nichol JL, Harker LA 1998 Ineffective platelet production in thrombocytopenic human immunodeficiency virus-infected patients. Blood 91:3239-3246

43. Tighe P, Rimsza LM, Christensen RD, Lew J, Sola MC 2005 Severe thrombocytopenia in a neonate with congenital HIV infection. J Pediatr 146:408-413 